\title{
Exploring Health System Responsiveness in Ambulatory Care and Disease Management and its Relation to Other Dimensions of Health System Performance (RAC) - Study Design and Methodology
}

\author{
Julia Röttger ${ }^{*}$, Miriam Blümel ${ }^{1}$, Susanne Engel ${ }^{2}$, Brigitte Grenz-Farenholtz ${ }^{2}$, Sabine Fuchs ${ }^{1}$, Roland Linder ${ }^{2}$, \\ Frank Verheyen ${ }^{2}$, Reinhard Busse ${ }^{1}$
}

\begin{abstract}
Background: The responsiveness of a health system is considered to be an intrinsic goal of health systems and an essential aspect in performance assessment. Numerous studies have analysed health system responsiveness and related concepts, especially across different countries and health systems. However, fewer studies have applied the concept for the evaluation of specific healthcare delivery structures and thoroughly analysed its determinants within one country. The aims of this study are to assess the level of perceived health system responsiveness to patients with chronic diseases in ambulatory care in Germany and to analyse the determinants of health system responsiveness as well as its distribution across different population groups.

Methods and Analysis: The target population consists of chronically ill people in Germany, with a focus on patients suffering from type 2 diabetes and/or from coronary heart disease (CHD). Data comes from two different sources: (i) cross-sectional survey data from a postal survey and (ii) claims data from a German sickness fund. Data from both sources will be linked at an individual-level. The postal survey has the purpose of measuring perceived health system responsiveness, health related quality of life, experiences with disease management programmes (DMPs) and (subjective) socioeconomic background. The claims data consists of information on (co)morbidities, service utilization, enrolment within a DMP and sociodemographic characteristics, including the type of residential area.

Discussion: RAC is one of the first projects linking survey data on health system responsiveness at individual level with claims data. With this unique database, it will be possible to comprehensively analyse determinants of health system responsiveness and its relation to other aspects of health system performance assessment. The results of the project will allow German health system decision-makers to assess the performance of nonclinical aspects of healthcare delivery and their determinants in two important areas of health policy: in ambulatory and chronic disease care.

Keywords: Data Linkage, Claims Data, Survey Data, Health System Responsiveness, Disease Management Programs, Patient Orientation

Copyright: @ 2015 by Kerman University of Medical Sciences

Citation: Röttger J, Blümel M, Engel S, et al. Exploring health system responsiveness in ambulatory care and disease management and its relation to other dimensions of health system performance (RAC) - study design and methodology. Int J Health Policy Manag. 2015;4(7):431-437. doi:10.15171/ijhpm.2015.97
\end{abstract}

Article History:

Received: 6 January 2015 Accepted: 12 May 2015 ePublished: 20 May 2015

\section{Background}

In 2000 the World Health Organisation (WHO) defined "responsiveness to citizens' legitimate expectations" as an intrinsic goal of health systems and, thus, as a main aspect in the performance assessment of health systems. ${ }^{1}$ In addition to the other dimensions of health systems performance assessment - health and fairness in financial contribution -, responsiveness refers to the nonclinical aspects of healthcare. It can further be subdivided into two major categories, each composing four domains: "Respect-for-persons" through dignity, confidentiality, autonomy, and communication; and "Client-orientation" consisting of social support, prompt attention, choice of care provider, and quality of basic amenities. ${ }^{1}$

The concept of health system responsiveness, mainly focusing on the comparison of health systems, has been used frequently in international studies, such as the Multi-Country
Survey Study on Health and Responsiveness (MCSS), ${ }^{2}$ the World Health Survey (WHS) 2002, ${ }^{3}$ the Survey of Health, Ageing and Retirement in Europe (SHARE) ${ }^{4}$ and the Study on Global Ageing and Adult Health (SAGE). ${ }^{5}$ Fewer studies applied the responsiveness concept for the evaluation of specific healthcare delivery structures or particular subgroups of the population and analysed its determinants within one country ${ }^{6-8}$ Moreover, to our knowledge, data on health system responsiveness has not yet been linked to claims data (eg, from sickness funds) or other routinely collected medical data on individual level, allowing for thorough analysis regarding (co)morbidities or the utilization of health services.

This manuscript presents the study design and methodology of the project "Exploring health system Responsiveness in Ambulatory Care and disease management and its relation to other dimensions of health system performance" (RAC). RAC is part of the Berlin Centre for Health Economics Research 
(BerlinHECOR), which has the overarching aim to further improve the methods for a comprehensive performance assessment of German healthcare. ${ }^{9}$ In this context, the project RAC focuses on two relevant aspects of health system assessment: the responsiveness of the health system to patients with chronic conditions and in ambulatory care settings. To allow for a thorough analysis of both aspects, the project follows two different approaches: in one study, general practitioners (GPs) and their respective patients will be surveyed; in the second, a survey is conducted of chronically ill patients in ambulatory care (GP and specialist care) and these survey data will subsequently be linked to individual level administrative claims data from a German sickness fund. The second study is carried out in close collaboration with the Scientific Institute of Techniker Krankenkasse for Benefit and Efficiency in Health Care (Wissenschaftliches Institut der Techniker Krankenkasse für Nutzen und Effizienz im Gesundheitswesen, WINEG). The following presentation of study design and methodology relates to the second approach.

In Germany, health insurance is mandatory for all residents. In $2014,85 \%$ of the population was covered under statutory health insurance (SHI) scheme (represented by 132 different sickness funds) and $11 \%$ were covered by substitutive private health insurance (PHI). Internationally compared, Germany spends many of its resources on health $(11.4 \%$ of Gross Domestic Product [GDP] in 2012) and has low costsharing. Insurants have access to a wide range of services and free choice of providers. ${ }^{10} \mathrm{~A}$ particular characteristic of the German health system is also the separation between the hospital sector, in which around 2000 hospitals still concentrate on providing inpatient care, and the ambulatory care sector, in which more than 120000 office-based physicians (the majority of them often single-handed) not only provide family medicine but also specialist care. ${ }^{10}$

This study not only aims to assess the level of health system responsiveness to patients with chronic diseases in ambulatory care in Germany, but also to scrutinize the determinants of health system responsiveness as well as the distribution of responsiveness across different population groups. This includes analyses on (1) differences between participants and nonparticipants of disease management programmes (DMPs) for the chronically ill, (2) differences among people from different residential areas (eg, rural vs. urban) and (3) differences among people with differing levels of service utilization (eg, high users vs. low users). Analyses will be based on a unique dataset - survey data on health system responsiveness linked to individual level administrative claims data from a German sickness fund - and will thus add important new aspects to the existing literature on health system responsiveness.

\section{Methods/Design}

The target population of the study is chronically ill people. With the aim of having homogenous patient groups, the study is split into 2 substudies both focusing on one specific (group of) chronic condition: One substudy focuses on type 2 diabetes and the other concentrates on coronary heart disease (CHD). These conditions were chosen for 3 reasons: (1) the high prevalence rates, (2) DMPs exist for both conditions, and
(3) the respective DMPs are highly utilized. ${ }^{11}$

The study is based on 2 different data sources: Survey data from a postal survey and administrative claims data from a German sickness fund (Techniker Krankenkasse, TK). We will use the benefits from both sources. To assess the perceived health system responsiveness of the chronically ill, we will conduct a postal survey. Furthermore, the survey data will be used to gain insight into the participants' health related quality of life, their experiences with DMPs and their (subjective) socioeconomic background (see Final Instrument section). Complementing the survey data, information on (co)morbidities, service utilization, enrolment within a DMP and/or integrated care programme, and sociodemographic characteristics, including the type of living area, will be obtained from SHI administrative claims data. The advantage of using SHI administrative claims data for this information is, among others, the avoidance of recall bias (eg, considering service utilization) and the avoidance of an overdemanding questionnaire (see SHI Administrative Claims Data section). Additionally, we will use the SHI claims data for the identification of our sample. For all planned data analyses, the data from both sources will be linked at individual level.

\section{Sample}

For each substudy (type 2 diabetes and CHD) we aim for a sample size of 2500 persons. Due to the planned linkage of survey and claims data, participants are not only asked to complete and return the questionnaire but to give their written informed consent for data linkage. Because experience with this study design is limited (with the linking of the data, asking participants to complete the questionnaire and to sign the consent form), we took a very conservative estimate of the response rate (between $10 \%$ to $15 \%$ ). Hence, the initial sample consisted of 26000 persons in each substudy. Eligible study participants were selected according to the following criteria. All persons were continuously insured at the TK from 1 January 2012 through 23 October 2013. In both substudies, 2 groups with 13000 participants each were generated.

1. One group of the insured who were continuously enrolled in the respective DMP (type 2 diabetes or CHD) from at least 1 January 2012 through 23 October 2013, and

2. A second group of the insured who suffer from type 2 diabetes and/or CHD, and fulfill the requirements but do not yet participate in one of the respective DMPs. Participants for both groups were selected randomly from the whole TK insured population that fulfilled the inclusion criteria. Table 1 depicts the inclusion and exclusion criteria. Study participants were selected for one of the substudies (eg, type 2 diabetes or CHD) only, ie, persons participating in both DMPs or who were eligible for both DMPs were included only in one substudy.

\section{Survey Data \\ Instrument Development}

On the basis of the WHO responsiveness concept, we conducted 4 focus groups with chronically ill people to test the concept's applicability for chronic disease care in Germany. The results from the focus groups indicated the overall suitability of the WHO responsiveness domains for 
Table 1. Inclusion and Exclusion Criteria

\begin{tabular}{lll}
\hline \multicolumn{1}{c}{ Inclusion } & \\
\hline - 18 to 90 years at date of initial sample selection (23.9.2013) & For All Groups & \\
& $\begin{array}{l}\text { Exclusion } \\
\text { Continuously insured at the TK (from 1.1.2012-23.10.2013) }\end{array}$ & $\begin{array}{l}\text { The insured with increased data security regulation (eg, former } \\
\text { employees of TK) }\end{array}$ \\
& $\begin{array}{l}\text { The insured who are unable to participate in a written survey } \\
\text { (eg, person with legal representative) } \\
\text { - Further TK standards (eg, insured person was contacted for a } \\
\text { different survey within last 12 months by TK) }\end{array}$
\end{tabular}

\begin{tabular}{|c|c|}
\hline \multicolumn{2}{|l|}{ DMP Participants } \\
\hline - Continuous enrollment in indication specific DMP (from 1.1.2012-23.10.2013) & \\
\hline \multicolumn{2}{|l|}{ Non-DMP Participants } \\
\hline $\begin{array}{l}\text { Minimum requirement for identification of type } 2 \text { diabetes patients based on claims } \\
\text { data: } \\
\text { - One selected ICD-code }{ }^{\text {a }} \text { from hospital care within last } 36 \text { months } \\
\text { OR } \\
\text { - One selected ICD-code from ambulatory care AND one selected ATC-code within } \\
\text { last } 12 \text { months } \\
\text { OR }\end{array}$ & - Enrolment in any other DMP (1.1.2012-23.10.2013) \\
\hline
\end{tabular}

Minimum requirement for identification of $\mathrm{CHD}$ patients based on claims data:

- One selected ICD-code from hospital care within last 36 months

- Enrolment in any other DMP (1.1.2012-23.10.2013)

OR

- One selected ICD-code from ambulatory care AND one of the selected ATC-codes

(level 1 or level 2) within last 12 months

OR

- One selected ATC-code (level 1) within last 12 months

Abbreviations: DMP, disease management programme; CHD, coronary heart disease; ATC, anatomical therapeutic chemical; ICD, International Classification of Diseases.

a See Table 2 for specific ICD and ATC codes.

the assessment of chronic care. Two further domains were identified for the measurement of the responsiveness of chronic disease care: coordination (coordination of course of treatment and among different healthcare providers) and trust (trust that treatment/consultation is not influenced by any motives other than the patients' well-being). ${ }^{12}$ Based on these results, and the related WHO questionnaires, which have been used within the MCSS and the WHS 2002 to assess the responsiveness of health systems, we developed the German-language survey questionnaire.

The developed questionnaire was tested qualitatively applying the Three-Step Test-Interview. ${ }^{13}$ We conducted cognitive interviews with 6 participants, all with chronic conditions and, thus, reflecting the target group of the postal survey. Overall, the respondents rated the questionnaire as clear and easy to understand and only slight modifications were necessary.

\section{Final Instrument}

The final questionnaire consists of 72 items. Table 3 depicts the topics in the same order as they appear in the questionnaire. The overall satisfaction with the healthcare system was operationalized using the question "All in all, how satisfied are you with your healthcare?"[1] with 5 answer categories ranging from "very satisfied" to "very unsatisfied."

The perceived health system responsiveness is the main dependent variable in the planned analyses. It is measured by 49 items in the questionnaire. The questions used in the short version MCSS questionnaire cover seven ${ }^{[2]}$ WHO domains of health system responsiveness. We used the original wording of the German version whenever possible and only made slight changes if necessary ${ }^{[3]}$. Based on the results of the focus groups and the pretest, we included ten additional items, both for the domains developed by the WHO as well as for the two additional domains (trust and coordination). All items were included twice: once to assess the responsiveness of the healthcare provided by the GP and once for specialist care. All included items had 5 answer categories ranging from "very good" to "very bad."

As a part of health system responsiveness, study participants were asked if they had experienced any kind of discrimination related to their healthcare within the last twelve months. In two following questions, participants could further specify in which aspect of their healthcare they perceived discrimination (eg, waiting times) and for which reason they thought they were disadvantaged (eg, no PHI).

Additionally, we assessed the level of unmet healthcare need by asking participants if they experienced any situation within the last twelve months in which they perceived a need for healthcare but did not seek healthcare. In two following questions, participants could further specify for which kind of healthcare (eg, specialist's consultation) and for which reason (eg, waiting times) they did not seek healthcare.

Like all questions asking for subjective ratings, the questions on health system responsiveness might be influenced by differences in answer behaviour among different population groups. One approach to control for these differences is the usage of anchoring vignettes. ${ }^{14,15}$ We thus included one anchoring vignette per domain (for logistical reasons it was not possible to include multiple vignettes per domain, which is the more preferable approach in the usage of anchoring vignettes).

We included the EQ-5D 5L to assess health-related quality of life, ${ }^{16}$ as we assumed an association between perceived wellbeing and perceived health system responsiveness. The EQ$5 \mathrm{D}$ is one of the most frequently used questionnaires to assess health-related quality of life and has been tested extensively 
Table 2. ICD and ATC Codes Used for Sample Identification

\begin{tabular}{ll}
\hline Type 2 Diabetes & \\
\hline ICD codes & E11-E14 \\
& A10AB01-A10AB05, A10AB30, A10AC01-A10AC04, A10AC30, A10AD01-A10AD05, A10AD30, A10AE01- \\
ATC codes & A10AE04, A10AE30, A10BA01-A10BA03, A10BB01-A10BB12, A10BB31, A10BC01, A10BD01-A10BD03, \\
& A10BF01-A10BF03, A10BG01-A10BG03, A10BH01, A10BP01, A10BP02, A10BP30, A10BX01-A10BX04, \\
\hline CHD & A10XA01 \\
\hline ICD codes & I20-I25, I50 \\
ATC-codes level 1 & C01DA02, C01DA05, C01DA08, C01DA14, C01DA52, C01DX11, C01DX12 \\
ATC codes level 2 & C07AA03, C07AA05, C07AA07, C07AB02, C07AB03, C07AB05, C07AB07, C07AB08, C07AB12, C07AB13, \\
\hline
\end{tabular}

Abbreviations: ATC, anatomical therapeutic chemical; ICD, International Classification of Diseases; CHD, coronary heart disease.

Table 3. Topics Covered by Questionnaire

\begin{tabular}{lc}
\hline Topic & No. of Items \\
\hline Overall satisfaction with healthcare system & 1 \\
Health system responsiveness & 17 \\
$\begin{array}{l}\text { Rated experiences with GP (related to last visit within } \\
\text { last } 12 \text { months) }\end{array}$ & 17 \\
$\begin{array}{l}\text { Rated experiences with specialist care (related to last } \\
\text { visit within last } 12 \text { months) }\end{array}$ & 3 \\
$\begin{array}{l}\text { Perceived discrimination within last } 12 \text { months } \\
\text { Perceived unmet need for healthcare within last } 12\end{array}$ & 3 \\
$\begin{array}{l}\text { months } \\
\text { Anchoring vignettes }\end{array}$ & 9 \\
Health related quality of life (EQ-5D 5L) & 6 \\
Questions on DMP & 7 \\
Sociodemographic characteristics & 7 \\
Subjective socioeconomic status & 1 \\
\hline Assistance with completing the questionnaire & 1 \\
\hline Abbreviations: DMP, disease management programme; GP
\end{tabular}

Abbreviations: DMP, disease management programme; GP, general practitioner.

regarding validity and reliability. ${ }^{17,18}$

As differences between participants and nonparticipants of DMPs are one focus of the project, we asked study participants if they take part in a DMP and in which of the 6 currently available DMPs they participate in. Information on enrolment in a DMP will also be available from claims data (see SHI Administrative Claims Data section). Hence, in the upcoming analysis, a differentiation between four groups of study participants will be possible: $(a)$ the person is enrolled in a DMP and knows about the enrolment, $(b)$ the person is enrolled in a DMP but does not know about the enrolment, (c) the person is not enrolled in a DMP but believes to be enrolled, $(d)$ the person is not enrolled in a DMP and knows that he/she is not enrolled. In addition, to analyse patient experiences with DMPs, we included six questions on DMPrelated patient education courses and documentation/ coordination within the DMP.

In addition, to questions related to 'objective' socioeconomic characteristics (gender, age, highest educational achievement, number of persons within household-in total and under 18 years-, monthly net-income), one item to assess the subjective socioeconomic status (subSES) was included. The subSES has been shown to correlate with health status. ${ }^{19}$ We thus hypothesized that it may also influence the perceived health system responsiveness. We operationalized subSES with the item "Today there is a lot of discussion about different social classes. In your opinion, which social class do you belong to?" [4]. The item was used previously in various surveys in Germany. ${ }^{20}$ However, based on the results from the qualitative pretest, we modified the answer categories slightly by changing 'working class' into 'lower middle class', which led to the following six answer categories: 'lower class,' 'lower middle class,' 'middle class,' 'upper middle class,' 'upper class,' and 'none of these.'

Since we expected our target group to be less healthy and older than the general population, we assumed that some of the surveyed persons might need help with completing the questionnaire. Hence, we asked all participants if they have had any assistance with filling in the questionnaire, to use this information as a control variable in the planned data analyses.

Statutory Health Insurance Administrative Claims Data Sickness funds routinely collect SHI claims data, which serves as a second data source for the purpose of this study. A German sickness fund - the TK - with 8.9 million insured people in 2014 provides claims data for this study. The data is used to derive information on morbidity, service utilization, and sociodemographic characteristics. Table 4 shows the topic related variables provided by $\mathrm{SHI}$ administrative claims data. Complementary to the subjective well-being assessed within the postal survey, information on disease severity as well as on further comorbidities is generated using claims data. For both substudies, type 2 diabetes and CHD, we selected disease specific ICD- (International Classification of Diseases), OPS(Operationen- und Prozedurenschlüssel - German procedure coding) and ATC-codes (Anatomical Therapeutic Chemical classification system), which represent differing levels of disease severity or frequent comorbidities. Additionally, as a generic comorbidity measure, we apply the Charlson comorbidity index for ambulatory care..$^{21,22}$

It is hypothesized that the structure of care delivery, which is operationalized by the status of DMP enrolment as well as the status of enrolment in an integrated care model (eg, family physician care model), determines the perceived health system responsiveness. Likewise, service utilization is one explanatory variable for the perception of health system responsiveness. It is operationalized for inpatient care by the number and length of hospital stays and for ambulatory care by the number of days with service accounting of GPs and specialists, both deduced from the claims data.

In Germany, fee-for-service is the regular payment system for GPs and specialists in ambulatory care. However, in 2009 a 
Table 4. Variables Derived From SHI Administrative Claims Data

\begin{tabular}{|c|c|c|}
\hline Topic & Variable & Indicator \\
\hline (Co)Morbidity & $\begin{array}{l}\text { Level of long-term care entitlement } \\
\text { Complications and comorbidities according to the } \\
\text { Charlson-comorbidity index and related to type } 2 \\
\text { diabetes and CHD }\end{array}$ & $\begin{array}{l}\text { Level } 1 / 2 / 3 \\
\text { Selected ICD-10 codes in ambulatory and hospital care, ATC codes, OPS codes }\end{array}$ \\
\hline Care delivery & $\begin{array}{l}\text { Enrolment in DMP } \\
\text { Enrolment in Integrated Care Model }\end{array}$ & $\begin{array}{l}\text { Enrolment in selected DMP and/or other DMP } \\
\text { Enrolment in Integrated Care Model }\end{array}$ \\
\hline Service utilization & $\begin{array}{l}\text { Number of hospital stays } \\
\text { Length of hospital stay } \\
\text { Contacts with ambulatory healthcare provider }\end{array}$ & $\begin{array}{l}\text { Number of admissions within one quarter } \\
\text { Total length of stay in days within one quarter } \\
\text { Number of days with service accounting separated by GP and specialist } \\
\text { within one quarter }\end{array}$ \\
\hline Sociodemographics & $\begin{array}{l}\text { Age } \\
\text { Gender } \\
\text { Living area }\end{array}$ & $\begin{array}{l}\text { In years } \\
\text { Male/female } \\
\text { Federal state, } 4 \text { and } 9 \text { categories of urbanization }\end{array}$ \\
\hline
\end{tabular}

Abbreviations: SHI, statutory health insurance; DMP, disease management programme; GP, general practitioner; CHD, coronary heart disease; ATC, anatomical therapeutic chemical; ICD, International Classification of Diseases; OPS, Operationen- und Prozedurenschlüssel.

quarterly capitation type lump sum has been introduced (paid if a patient has at least one contact) which includes several usually provided services (eg, medical care, coordination, documentation). Thus, the exact number of contacts with GPs and specialists cannot-be derived from claims data, and we use the number of days with service accounting as a proxy. This may lead to an underestimation of actual contacts, since some of these do not lead to separate accounting, but fall under the lump sum.

Healthcare service provision can be assumed to vary strongly between urban and rural living areas; perceived health system responsiveness may vary accordingly. Due to data protection regulations, the inclusion of the postal or district code in the final dataset was impossible. Yet, it was possible to transfer the district codes into 4 and 9 categories, respectively, that describe the level of urbanization according to the categorization system of the German Federal Institute for Research on Building, Urban Affairs and Spatial Development. To allow for sensitivity analysis, we transferred the codes to both systems. Additionally, information on the federal state of the residential area is available.

\section{Sample and Data Linkage}

For all selected participants, a pseudonym was used. Participants received the described questionnaire and a letter of informed consent (both with their pseudonym) as well as information on data protection. In the letter of informed consent, participants agreed to the linkage of selected individual-level SHI administrative claims data with their respective survey data. Only data of participants who completed the questionnaire and signed the informed consent will be included in further analyses (see Figure). Via the pseudonyms, the survey data will be linked with the claims data at individual-level. Pseudonyms will be deleted after the linkage to generate anonymous data.

While the questionnaire was mailed at the end of October 2013, it included several questions relating to the last 12 months (eg, the questions on health system responsiveness). The SHI administrative claims data cover 6 quarters, from April 2012 through September 2013, and hence overlap with the retrospective survey data.

\section{Data Cleaning and Planned Analysis}

All analyses will be conducted on a cleaned dataset with cases that fulfil the following minimum requirements regarding data quality. As all planned analyses need information from both data sources, only the cases with linked questionnaire and claims data will be included. Questionnaire data will be excluded if parts from the questionnaire (eg, whole pages, or cut-outs from pages) are missing. In addition, if more than $50 \%$ of the questionnaire pages are not completed, or if systematically the front- or the backside from the questionnaire is not filled-in, cases will also be excluded. As all planned analyses will include information on the sociodemographic characteristics provided in the questionnaire, cases without any of this information will also be excluded.

The planned data analyses incorporate various strategies: these include descriptive analyses, multivariate analyses as well as the modelling of heterogeneous reporting behaviour based on answers to anchoring vignettes. Special attention will be paid to the comparison of different groups, eg, DMP-participants and nonparticipants and suitable methods will be applied to account for group differences. Differences in reporting behaviour regarding the health system responsivenessitems will be analysed by means of the included anchoring vignettes. $^{4}$

\section{Preliminary Results and Feasibility}

A total sample of 51998 persons was contacted. Of these, $17213(33.10 \%)$ sent back the questionnaire and 16672 $(32.06 \%)$ sent back the informed consent, which makes a gross response rate of $33.29 \%$ ( 17311 individual cases). Of 16573 (31.87\%) linked cases, 1008 cases were excluded due to inadequate completed questionnaire and after plausibility check. The final response of valid cases is $n=15565$ (29.93\%). Table 5 shows the results from the (non)responder analysis. In all categories, patients with diabetes show a lower response rate than patients with CHD. In both subgroups men and patients enrolled in a DMP have a higher response rate than women and patients not enrolled in a DMP, respectively. Furthermore, the response rate increases with age but decreases in the age group of $>80$ years. The response rate across the federal states ranges between $40.99 \%$ in Saxony 
and $25.90 \%$ in Mecklenburg-West Pomerania (CHD) and between 34.84\% in Saxony and 21.43\% in Mecklenburg-West Pomerania (Diabetes).

\section{Data Protection}

Since this study includes a survey of statutory health insured, who were contacted by their sickness fund, certificate of nonobjection for the study was obtained by the German Federal (Social) Insurance Authority.

The project partners, in accordance with the respective data security engineers, have developed strict criteria (included in the project proposal) regarding the sampling of patients, the mailing of the questionnaire, and the storage, linkage and access of the data to secure the privacy and confidentiality of

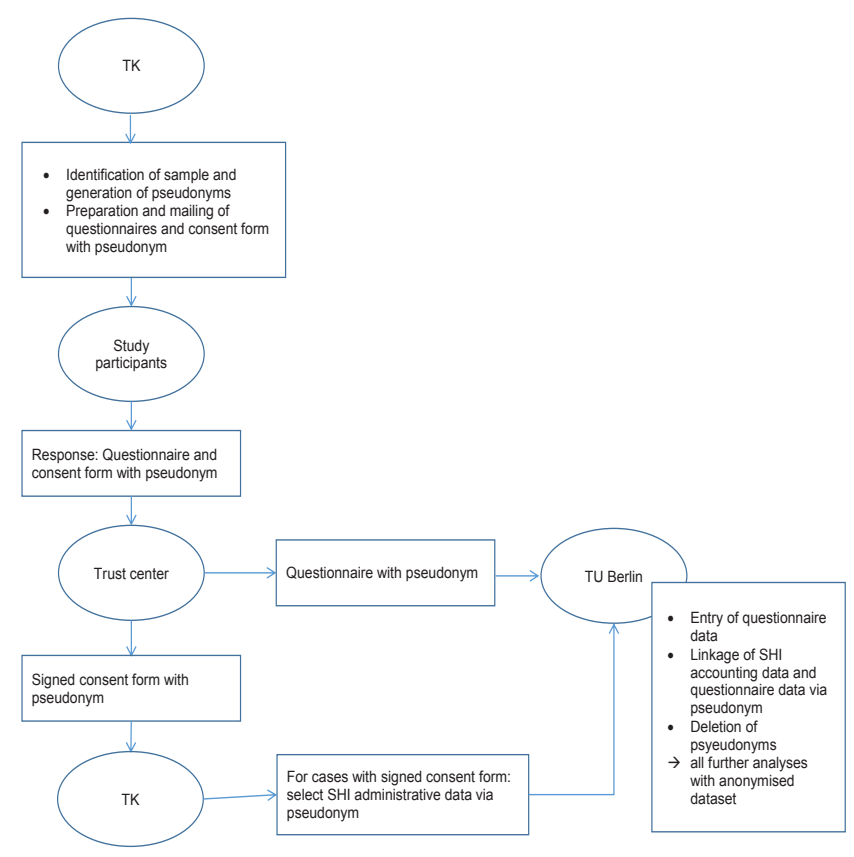

Figure. Linkage of Survey and statutory health insurance (SHI) Administrative Claims Data. the data at all times during the project.

\section{Discussion}

To our knowledge, this is the first study linking survey data to health system responsiveness at individual level with SHI administrative claims data, comprising information on medical diagnoses, prescriptions of pharmaceuticals, healthcare service utilization and sociodemographic characteristics.

With this unique database, we will be able to comprehensively analyse determinants of health system responsiveness and its relation to other aspects of health system performance assessment, such as health outcomes and equity. Yet, it has to be considered that, due to the nature of the data (only from one sickness fund, only specific diseases), the results from the project will not be necessarily generalizable for the German healthcare system as a whole. Nevertheless, the project will lead to new insights on the determinants of health system responsiveness, eg, with regard to service utilisation and (co) morbidities. In addition, it will allow German health system stewards to assess the performance of nonclinical aspects of healthcare delivery and their determinants in two important areas of health policy: in outpatient and chronic disease care. The usage and linkage of different data sources is of high potential for assessing health system performance not only in Germany but internationally. Yet, there are still various aspects in health system performance assessment where the combination of various datasets has hardly been explored or actually applied. In this study protocol we demonstrate one potential approach for using different data sources in health system performance assessment. We use SHI administrative claims data for the sample selection and link them eventually to survey data, which imposes a particular challenge to the conceptualization of data protection requirements as well as the sample selection. Even though the approach was related to a rather high effort for the survey participants (completion of the questionnaire and signing the informed consent form) the preliminary results with a net-response rate of $29.93 \%$ indicate

Table 5. Sample Response

\begin{tabular}{|c|c|c|c|c|c|c|}
\hline & \multicolumn{3}{|c|}{ CHD } & \multicolumn{3}{|c|}{ Diabetes type 2} \\
\hline & Original Sample (n) & Finally Linked (n) & Response Rate (\%) & Original Sample (n) & Finally Linked (n) & Response Rate (\%) \\
\hline \multicolumn{7}{|l|}{ Gender } \\
\hline Female & 7959 & 2131 & 26.77 & 9691 & 2326 & 24.00 \\
\hline Male & 18040 & 6345 & 35.17 & 16308 & 4763 & 29.21 \\
\hline \multicolumn{7}{|l|}{ Age } \\
\hline$\leq 60$ years & 6245 & 1292 & 20.69 & 8521 & 1485 & 17.43 \\
\hline $61-65$ years & 3185 & 902 & 28.32 & 3852 & 1044 & 27.10 \\
\hline $66-70$ years & 3546 & 1216 & 34.29 & 3831 & 1182 & 30.85 \\
\hline $71-75$ years & 5547 & 2220 & 40.02 & 4690 & 1645 & 35.07 \\
\hline $76-80$ years & 4280 & 1763 & 41.19 & 3246 & 1186 & 36.54 \\
\hline$>80$ years & 3196 & 1083 & 33.89 & 1859 & 547 & 29.42 \\
\hline \multicolumn{7}{|l|}{ DMP enrolment } \\
\hline Yes & 13000 & 5141 & 39.55 & 13000 & 4228 & 32.52 \\
\hline No & 12999 & 3335 & 25.66 & 12999 & 2861 & 22.01 \\
\hline \multicolumn{7}{|l|}{ Region } \\
\hline West-Germany & 20534 & 6608 & 32.18 & 20154 & 5399 & 26.79 \\
\hline East-Germany & 5465 & 1867 & 34.16 & 5845 & 1690 & 28.91 \\
\hline Total & 25999 & 8476 & 32.60 & 25999 & 7089 & 27.27 \\
\hline
\end{tabular}

Abbreviations: DMP, disease management programme; $\mathrm{CHD}$, coronary heart disease. 
a good acceptance of the approach by the survey participants. Thus, this approach should be of interest for health system performance assessment in general.

\section{Acknowledgements}

We would like to thank the following persons for their valuable contributions: Philipp Grevenstette, Katharina Hinsch, Natalia Kelsch, Angela Kloppenburg, Julia Köppen, Daria Langner, Frauke Schulz, Caroline Wagner, all participants of the focus groups and all survey participants.

The project was funded as part of the Berlin Centre for Health Economics Research by the German Federal Ministry of Education and Research, Bonn, Germany (grant no. 01EH1202A).

\section{Ethical issues}

The project has been granted financial support from the German Federal Ministry of Education and Research, Bonn, Germany. The study protocol was approved by the ethical review board of the Technische Universität Berlin, Berlin, Germany. Proof of the ethical approval has been submitted to the editorial board of this journal for verification.

\section{Competing interests}

$\mathrm{MB}, \mathrm{RB}, \mathrm{SF}$, and JR declare that they have no competing interests. FV, RL, SE, and BGF work at the Scientific Institute of TK for Benefit and Efficiency in Health Care. They declare that they have no competing interests.

\section{Authors' contributions}

$\mathrm{JR}$ and MB had the main role in manuscript preparation. RB and JR had the main responsibility for developing the research question. RB, JR, and FV had the main responsibility for developing the study design. JR, SF, and MB together with BGF prepared the survey data collection, while RL and SE were responsible for the $\mathrm{SHI}$ administrative claims data query. JR, MB, and SF will be responsible for the upcoming statistical analyses. All those listed as authors were responsible for reading, commenting upon, and approving the final manuscript.

\section{Authors' affiliations}

${ }^{1}$ Department of Health Care Management, Berlin Centre for Health Economics Research, Technische Universität Berlin, Berlin, Germany. ${ }^{2}$ Wissenschaftliches Institut der TK für Nutzen und Effizienz im Gesundheitswesen (WINEG), Hamburg, Germany.

\section{Endnotes}

[1] Please note that the original questionnaire is in German.

[2] We excluded the domain "social support" as it is only used for inpatient care. [3] That is: we made changes to the "prompt attention" item, as we differentiate between the waiting time in the waiting room and the waiting time from asking for an appointment until the appointment; we made slight changes in the "autonomy" item by replacing "the treatment" with "your treatment."

[4] Authors' own translation, original wording in German.

\section{References}

1. Murray CJ, Frenk J. A framework for assessing the performance of health systems. Bull World Health Organ. 2000;78:717-731. doi:10.1590/S0042-96862000000600004

2. Üstün, TB, Chatterji S, Villanueva M, et al. WHO Multi-country Survey Study on Health and Responsiveness 2000-2001. In: Murray CJ, Evans DB, eds. Health System Performance Assessment: Debates, Methods and Empiricism. Geneva: World Health Organization; 2003:761-796.

3. Üstün TB, Sonmath C, Abdelhay M, Murray CJL, WHS Collaborating Group. The World Health Surveys. In: Murray CJ, Evans DB, eds. Health System Performance Assessment: Debates, Methods and Empiricism. Geneva: World Health Organization; 2003:797-808.

4. Sirven N, Santos-Eggimann B, Spagnoli J. Comparability of health care responsiveness in europe. Soc Indic Res. 2011;105:255271. doi:10.1007/s11205-011-9880-z

5. Rockers PC, Kruk ME, Laugesen MJ. Perceptions of the health system and public trust in government in low- and middle-income countries: evidence from the World Health Surveys. $J$ Health Polit Policy Law. 2012;37:405-437. doi:10.1215/03616878-1573076

6. Bramesfeld A, Wedegärtner $F$, Elgeti H, Bisson S. How does mental health care perform in respect to service users' expectations? Evaluating inpatient and outpatient care in Germany with the WHO responsiveness concept. BMC Health Serv Res. 2007;7:99. doi:10.1186/1472-6963-7-99

7. Liabsuetrakul T, Petmanee P, Sanguanchua S, Oumudee N. Health system responsiveness for delivery care in southern Thailand. Int J Qual Health Care. 2012;24:169-175. doi:10.1093/ intqhc/mzr085

8. Peltzer K, Phaswana-Mafuya N. Patient experiences and health system responsiveness among older adults in South Africa. Glob Health Action. 2012;5:1-11. doi:10.3402/gha.v5i0.18545

9. Busse R, Siegel M, Sundmacher L. Gesundheitsökonomisches Zentrum Berlin (Berlin HECOR). Public Health Forum. 2013;21:22. e1-22.e3. doi:10.1016/j.phf.2013.09.009

10. Busse R, Blümel M. Germany: health system review. Health Systems in Transition. 2014;16:1-296.

11. Bundesversicherungsamt. Zulassung der strukturierten Behandlungsprogramme (Disease Management Programme - DMP) durch das Bundesversicherungsamt (BVA). http:// www.bundesversicherungsamt.de/druckversion/weiterethemen/disease-management-programme/zulassung-diseasemanagement-programme-dmp.html\#c204. Updated December 2014. Accessed Jan 4, 2015.

12. Röttger J, Blümel $M$, Fuchs $S$, Busse $R$. Assessing the responsiveness of chronic disease care - Is the World Health Organization's concept of health system responsiveness applicable? Soc Sci Med. 2014;113:87-94. doi:10.1016/j. socscimed.2014.05.009

13. Hak T, van der Veer $\mathrm{K}$, Jansen $H$. The Three-Step TestInterview (TSTI): an observation-based method for pretesting self-completion questionnaires. Survey Research Methods. 2008;2:143-150.

14. King G, Murray CJL, Salomon JA, Tandon A. Enhancing the validity and cross-cultural comparability of measurement in survey research. Am Pol Sci Rev. 2004;98:191-207. doi:10.1017/ S000305540400108X

15. Murray CJ, Özaltin E, Tandon A, Salomon JA, Sadana R, Chatterji S. Empirical evaluation of the anchoring vignette approach in health surveys. In: Murray CJ, Evans DB, eds. Health System Performance Assessment: Debates, Methods and Empiricism. Geneva: World Health Organization; 2003:369-399.

16. Agborsangaya $C B$, Lahtinen $M$, Cooke $T$, Johnson JA. Comparing the EQ-5D $3 \mathrm{~L}$ and $5 \mathrm{~L}$ : measurement properties and association with chronic conditions and multimorbidity in the general population. Health Qual Life Outcomes. 2014;12:74. doi:10.1186/1477-7525-12-74

17. Kind P, Brooks R, Rabin R. EQ-5D concepts and methods: a developmental history. Dordrecht: Springer; 2005.

18. Rabin R, de Charro F. EQ-5D: a measure of health status from the EuroQol Group. Ann Med. 2001;33:337-343.

19. Hegar R, Mielck A. "Subjektiver sozialer Status". Prävention und Gesundheitsförderung 2010;5:389-400. doi:10.1007/s11553010-0261-2

20. Hegar R, Döring A, Mielck A. Relevance of ,subjective social status' for health risks and health status - results from the KORAF4-study [in German]. Gesundheitswesen. 2012;74:306-314. doi:10.1055/s-0031-1283551

21. Charlson ME, Pompei P, Ales KL, MacKenzie CR. A new method of classifying prognostic comorbidity in longitudinal studies: development and validation. J Chron Dis. 1987;40:373-383. doi:10.1016/0021-9681(87)90171-8

22. Charlson ME, Charlson RE, Peterson JC, Marinopoulos SS, Briggs WM, Hollenberg JP. The Charlson comorbidity index is adapted to predict costs of chronic disease in primary care patients. J Clin Epidemiol. 2008;61:1234-1240. doi:10.1016/j. jclinepi.2008.01.006 